\title{
Florid vascular proliferation in repeated intussusception mimicking primary angiomatous lesion
}

\author{
K L Ramsden, J Newman, A Moran
}

\begin{abstract}
A case of recurrent intussusception in a 76 year old man associated with vascular proliferation is reported. The initial biopsy specimen showed that ulceration and inflammation were not features. The proliferation was so florid as to point to an angiomatous lesion. At this stage a diagnosis of intussusception was not considered. The clinical impression was that of a caecal mass associated with a filling defect visible on barium enema and a fleshy "suspicious" lesion on colonoscopy.

This case illustrates the possibility of misinterpreting the importance of florid vascular proliferation in biopsy material where other features indicative of a reparative process are absent.
\end{abstract}

(F Clin Pathol 1993;46:91-92)

\section{Case report}

A 76 year old man presented with epigastric discomfort. Investigations revealed oesophageal reflux and a hiatus hernia and he was treated accordingly. Coincidentally, a vague mass was noted in the right iliac fossa, on two of several outpatient visits. A barium enema showed a filling defect in the caecum with dilatation of the ileo-caecal valve and terminal ileum. In view of these findings a colonoscopy was performed at which a $3 \mathrm{~cm}$ "suspicious"looking, irregular fleshy lesion was seen in the wall of the caecum. Above this, a benign looking polyp was present at the caecal pole. Biopsy specimens of the "suspicious" lesion were taken. He had never been treated with non-steroidal anti-inflammatory drugs (NSAIDS) nor potassium tablets and was not anaemic. Two months later a laparotomy was performed: a $10 \mathrm{~cm}$ ileo-caecal intussusception, which was reduced, was found and a right hemicolectomy performed. In retrospect he had been asymptomatic with regard to the intussusception, and furthermore, both at the time of colonoscopy and barium enema, an intussusception was present but was not recognised as such. The benign-looking polyp, initially seen at colonoscopy, was still present and was leading the intussusception. He made an uneventful recovery.

\section{Pathological findings}

The biopsy material comprised superficial, flat, and papillary mucosa containing many small, thin-walled vascular channels within the lam- ina propria. The surface epithelium was singlelayered, intact though thin, and inflammation was not a feature. The vascular proliferation was so pronounced, and especially in the absence of inflammation, raised the possibility of a primary angiomatous lesion (fig 1). The clinical information of a localised "suspicious" lesion further supported this possibility.

The subsequent hemicolectomy specimen consisted of $23 \mathrm{~cm}$ of terminal ileum and $17 \mathrm{~cm}$ of caecum and ascending colon with an attached appendix. Eight centimetres from the proximal resection margin was a $2.5 \mathrm{~cm}$ smooth mucosal covered polyp consisting of fat. Adjacent and distal to the polyp, the mucosa was raised and irregular (the site previously biopsied) and the ileo-caecal valve was oedematous (fig 2).

Microscopic examination of the leading

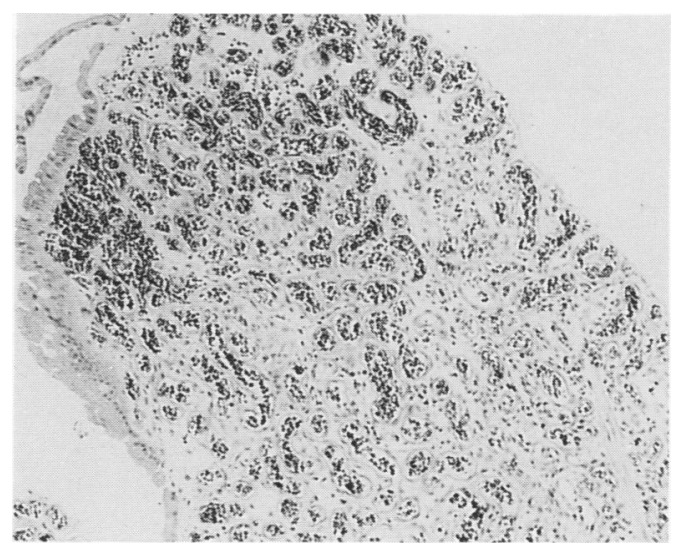

Figure 1 Noticeable vascular proliferation in the absence of inflammation or surface ulceration.

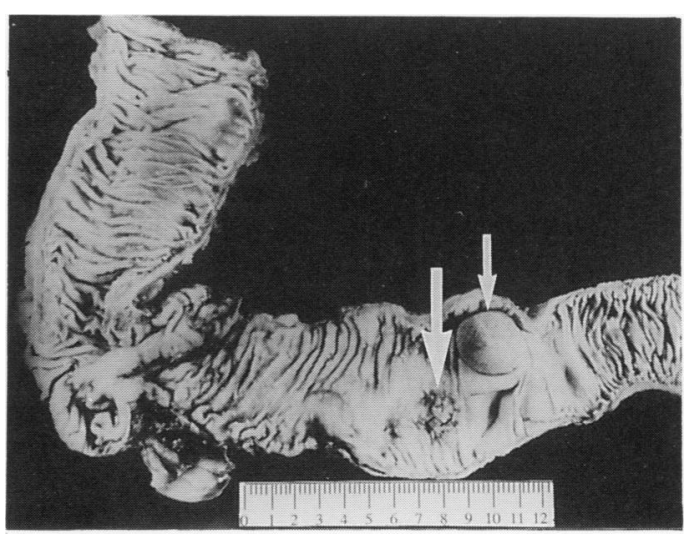

Figure 2 Right hemicolectomy specimen showing lipomatous polyp (small arrow), with irregular mucosa distal and adjacent (large arrow) to the polyp. This is the area from which the previous biopsy specimen was taken. 
polyp showed it to be a submucosal lipoma. Ileo-caecal oedema was confirmed. Adjacent to the polyp the raised and irregular mucosa showed moderate inflammation, and areas of ulceration and epithelial regeneration. There was also florid vascular proliferation throughout the mucosa in this region, similar to that seen in the biopsy specimen, with extension of the process into the submucosa with associated mild fibrosis. These findings suggested a reparative process, ischaemic damage due to the effects of intussusception being the most likely event.

\section{Discussion}

It is well recognised that ischaemic damage to mucosa and submucosa may occur in intussusception as a result of compression of the blood supply. ${ }^{1}$ In the acute phase of ischaemic damage the mucosa shows necrosis with overlying fibrin. ${ }^{2}$ In the subacute phase there is proliferation of capillaries in the form of granulation tissue, usually associated with ulceration and inflammation. ${ }^{3}$ In the chronic phase there is, in addition, fibrosis of the lamina propria. ${ }^{4}$

In the absence of an intussusception, the features seen in the biopsy specimen could theoretically represent a reparative process following simple ulceration. The localised nature and site of the lesion would make inflammatory bowel disease an unlikely cause. Similarly, the site of the lesion would make a drug induced ulceration unlikely and there was also no history of ingestion of any drug that could induce ulceration. Other intestinal lesions to be considered in the differential diagnosis of vascular proliferation involving thin-walled small vessels included angiodys- plasia (vascular ectasias) and Kaposi's sarcoma. Angiodysplasia is characterised by the presence of thin-walled vascular channels which tend to be dilated, vary in size, and extend primarily from the submucosa into the mucosa. Although subsequent ulceration could result in associated reparative features, the patient was not anaemic and there was no history of gastrointestinal haemorrhage. In Kaposi's sarcoma there is a proliferation of densely packed spindle shaped cells, some with atypical nuclei, producing small slit-like lumina within vascular spaces containing red cells. The lesion is primarily submucosal but may extend to involve the mucosa.

Biopsy material taken from an area of intestinal mucosa experiencing intermittent ischaemia may present with pronounced vascular proliferation in the absence of other features indicative of a reparative process. The appearance is reminiscent of a pyogenic granuloma. Such changes could lead to misinterpretation of biopsy material, especially if biopsy specimens are small and superficial and the clinical history, radiological studies, and colonoscopy do not raise the suspicion of ischaemia or intussusception.

1 Thomas Norris H. Re-examination of the Spectrum of Ischaemic Bowel Disease. In: Pathology of the colon, small
intestine and anus. New York: Churchill Livingstone, intestine and

2 Talbot K, Price AB. Ischaemic colitis. In: Biopsy pathology in colorectal disease. London: Chapman and Hall Ltd, 1987:189-94.

3 Gratama S. Vascular disorders, abnormalities, ischaemia, vasculitis. The large intestine. In: Whitehead $R$, ed. Gastrointestinal and oesophageal pathology. Edinburgh: Churchill Livingstone, 1989:556-94.

4 Morson BC, Dawson MP. Vascular disorders, Histopathology of intestinal ischaemia. In: Gastro-intestinal pathology. 2nd Edn. Oxford: Blackwell Scientific Publications, 1979:385-88. 